\title{
Efficacy of biodegradable osteofixation devices in oral and maxillofacial surgery remains inconclusive
}

\author{
Is there a difference in stability and morbidity with fixation of bone segments with \\ biodegradable or titanium fixation devices in orthognathic and trauma surgery?
}

\author{
Buijs GJ, Stegenga B, Bos RRM. Efficacy and safety of biodegrad- \\ able osteofixation devices in oral and maxillofacial surgery: a sys- \\ tematic review. J Dent Res 2006; 85:980-989
}

Data sources Medline (1966-2005), Embase (1989-2005) and (CENTRAL) Cochrane Central Register of Controlled Trials (1800-2005) were searched. Several experts were contacted to ensure that eligible studies were not overlooked. Key journals were searched by hand, together with reference lists of all relevant articles. No language restrictions were applied.

Study selection For inclusion, an article had to satisfy the following criteria. It should be a controlled clinical trial, and there should be union/ non-union of fracture or osteotomy in the maxillofacial skeleton; the presence of wound healing/ infection; intervention with a biodegradable or titanium osteofixation device; well-established (by clinical and radiographic evaluation) diagnoses and indications for treatment; and a follow-up period of $>6$ months.

Data extraction and synthesis Eligible studies were independently evaluated by two assessors using a quality assessment scale which was developed by Sindhu et al. ${ }^{1}$ The scale consists of 53 items in 15 dimensions. Agreement regarding the weighting of the individual subdimensions and the required minimum values for each dimension was reached in a consensus meeting.

Results The search identified 240 articles, of which five met the inclusion criteria. Three studies were randomised controlled trials whereas the other two were quasi-randomised. There was no significant difference between biodegradable and titanium osteofixation devices with regard to short-term outcome, complication rate and infections in orthognathic surgery. Regarding the fixation of traumatically fractured bone segments, no firm conclusion could be drawn because of the lack of controlled clinical trials. It was not possible to perform a meta-analysis because of the different outcomes used in the studies.

Conclusions Definite conclusions regarding the long-term performance of biodegradable fixation devices used in maxillofacial surgery cannot be drawn.

\section{Commentary}

As noted in this review under "General aspects of bone surgery," rigid internal fixation (RIF) is a major breakthrough in maxillofacial trauma and reconstructive surgery. RIF using plates and screws allows immediate function after repairing facial fractures and reconstructive operations. No longer do "jaws need to be wired shut" for fixation (maxillomandibular fixation). Advantages of RIF include early return to function and work, and shorter length of hospital stays. Tangible disadvantages include the increased cost of materials and operating time, and the possible need for removal of plates and screws. Theoretical disadvantages include a possible mutagenic effect of titanium, although there is no evidence for this in human clinical studies.

RIF plates and screws made of biodegradable materials are effective in achieving RIF and early return to function and have the obvious advantage of degrading. In animal and human studies, however, degradation is often incomplete and the residual plate material may be associated with infection or inflammation necessitating removal.

There is some controversy over the use of plates and screws made of titanium (the current gold standard for fixation materials) versus biodegradable materials. The authors state that, "the major drawback to the general use of biodegradable devices is the lack of clinical evidence." I disagree with this reasoning. For better or worse, clinicians commonly use devices and techniques without good clinical evidence. Instead, the handling properties and application of currently available biodegradable fixation devices, compared with titanium, are sufficiently poor and awkward to impede their general distribution alongside their lack of clear, unambiguous clinical or biological advantages. As the authors show in their well-conducted review of the topic, neither short- nor long-term advantages of biodegradable materials are at all apparent.

I do agree with the authors' statement that another significant reason for the limited use of biodegradable RIF devices, "is surgeons' resistance to modify the conventional treatment techniques with which they have the most experience." Surgeons, however, will quickly convert to new techniques given clear advantages of the technique or material. Most maxillofacial surgeons currently in practice have adopted the use of RIF (with titanium or biodegradable devices). We now rarely wire the teeth together for fixation despite our comfort and extensive experience with the latter operation.

For those interested in a current assessment of titanium and biodegradable materials in the clinical setting of maxillofacial fixation, I recommend this excellent review.

\section{Tom Dodson}

Harvard School of Dental Medicine, Boston, Massachusetts, USA

1. Sindhu F, Carpenter L, Seers K. Development of a tool to rate the quality assessment of randomized controlled trials using a Delphi technique. J Adv Nurs 1997; 25: 1262-1268.

Evidence-Based Dentistry (2007) 8, 5-6. doi:10.1038/sj.ebd.6400491
Address for correspondence: GJ Buijs, Department of Oral and Maxillofacial Surgery, University Medical Centre Groningen, University of Groningen, Hanzeplein 1, PO 30.001, 9700 RB Groningen, The Netherlands. E-mail: g.j.buijs@kchir.umcg.nl 